\title{
Maximisation of Correct Handover Probability and Data Throughput in Vehicular Networks
}

\author{
L. Banda and M. Mzyece \\ Department of Electrical Engineering and The French South African Institute of \\ Technology (F'SATI), Tshwane University of Technology, Gauteng, \\ South Africa
}

Received 15 April 2014; Accepted 24 May 2014

Publication 4 August 2014

\begin{abstract}
In the past decade, the networking and automobile industry has experienced the emergence of vehicular networks which were developed under the Intelligent Transportation Systems (ITS) to provide a plethora of safety and non-safety related applications. The provision of seamless mobility and session continuity is one of the major challenges for the transmission of ITS applications in vehicular networks. This is more critical when a communicating node moves from one subnet to another, a process referred to as inter-subnet handover. In such a case, we deal with the problem of fast and seamless handover support for better Quality of Service (QoS) provisioning especially, for throughputsensitive and delay-intolerant ITS applications. In this paper, we propose a scheme aimed at improving the handover performance in IP-based vehicular networks by maximising the correct handover probability $\left(P_{\text {Correct }}\right)$ and increasing the data throughput during inter-subnet handovers. We demonstrate the impact of mobile node's speed and direction of motion on both $P_{\text {Correct }}$ and data throughput via numerical analysis.
\end{abstract}

Keywords: Handover, Internet Protocol (IP), Correct Handover Probability, Data Throughput, Vehicular Networks.

Journal of Machine to Machine Communications, Vol. 1, 123-144.

doi: 10.13052/jmmc2246-137X.123

(C) 2014 River Publishers. All rights reserved. 


\section{Introduction}

Wireless vehicular communication networks were developed under the Intelligent Transportation System (ITS) technology to improve the safety, efficiency and environmental sustainability of transportation systems. ITS applications can be classified into two broad categories: safety and non-safety applications. Safety applications are concerned with sharing of information on accidents, weather forecast, traffic congestion, road works and other precautionary measures within and among communicating vehicles. On the other hand, non-safety applications involve infotainment services like on-board Internet access, instant messaging, remote access of servers, electronic toll payments and so forth $[1,2]$.

Vehicular networks are an emerging ITS technology integrating wireless communication into the automobile industry. As a result, different standardisation bodies (e.g., IEEE and IETF) have been working in collaboration with various consortia (e.g., Car-to-Car Communications Consortium (C2C-CC [3]) on several issues concerning vehicular communications networks. Generally, vehicular networks consist of two types of wireless communication nodes which are Dedicated Short Range Communication Devices (DSRC). These are: On-Board Units (OBUs) mounted on vehicles and interlinked with Application Units (AUs) such as laptops, tablets and smart phones used by passengers; and Road Side Units (RSUs) found on fixed network infrastructure such as cellular Base Stations (BSs) or WiFi Physical Hot Spots (PHSs). The main modes of wireless communication present in vehicular networks are: vehicle-to-vehicle $(\mathrm{V} 2 \mathrm{~V})$, vehicle-to-roadside (V2R) and inter-roadside (R2R) communication. Figure 1 shows a typical vehicular network architecture.

In wireless communication networks, handover management and location management are the two basic functionalities performed by the mobility management protocols for seamless mobility to be achieved. Handover management means keeping communication between two nodes alive as the Mobile Node (MN) moves freely and changes its point of attachment to the network. On the other hand, location management involves identifying the current location of an $\mathrm{MN}$ and keeping track of its location changes as it moves within the network [4]. Mobility management is vital in IP-based wireless networks, particularly during inter-subnet handovers when an MN changes its point of attachment to the Internet. For IP session continuity to be maintained during inter-subnet handovers, the IP-layer mobility management protocols should be able to support the rapid wireless link changes and the 


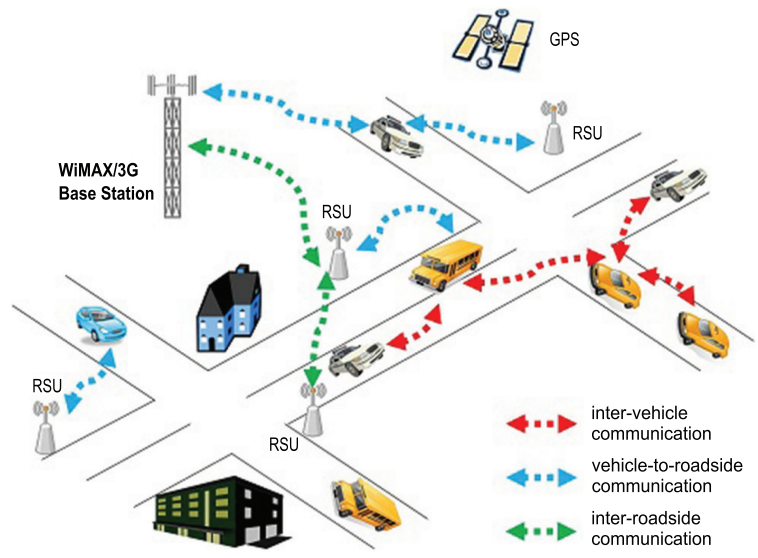

Figure 1 Typical vehicular network architecture [3]

fast IP configuration procedures [2, 5]. However, most current IP mobility management schemes fall short in that respect and as a consequence, a degradation in the Quality of Service (QoS) is registered for most applications.

The movement pattern of vehicles plays a critical role in the modelling and performance analysis of wireless IP networks. This study involves vehicle-toroadside infrastructure (i.e. V2I) communication and focuses on network layer inter-subnet handovers when a single $\mathrm{MN}$ changes its point of attachment to the global Internet. The large signalling delays associated with inter-subnet handovers can be detrimental to throughput-sensitive and delay-intolerant ITS applications [6, 7]. In this paper, we propose a mobility model aimed at reducing the signalling delay thereby, maximising the probability of correct handover initiation and increasing the data throughput during inter-subnet handovers in wireless vehicular scenarios.

The remainder of the paper is organised as follows. Section 2 presents the background and the related work found in the literature. Section 3 provides a system description and modelling of the proposed scheme. Section 4 presents the simulations methodology and numerical analysis. Section 5 finally, concludes the paper.

\section{Background and Related Work}

Mobility models are used to represent the movement patterns of mobile users in a network. They are employed to document how the MN's location, velocity and direction of motion change with time. Various approaches can 
be adopted in modelling the movement of vehicles, and they all undergo a common trade-off between precision and complexity. The research community in both industry and academia have devoted considerable efforts in their quest to address the problem of severe signalling overheads and inaccurate mobility modelling which lead to miss-allocation of the limited radio resources during inter-subnet handovers. To this end, numerous standard and non-standard mobility models have been outlined in the literature [8].

\subsection{Standard Vehicular Mobility Models}

From the analytical and mathematical point of view, the following mobility models have been standardised for the wireless vehicular environment.

\section{Constant Speed Motion (CSM) Model}

This model describes a random vehicular movement on a graph, representing a road topology. No particular constraint is forced on the graph nature so as to display different levels of realism [9]. The vehicle's motion is structured in movements between vertices of the graph referred to as destinations which are randomly selected. At the start of each trip, a vehicle $i$ chooses its next destination, computes the route to it by running a shortest path algorithm on the graph with link costs possibly influenced by parameters such as road length, speed limits, traffic congestion and so forth. The vehicle sets its speed to a value given by

$$
v_{i}=v_{\min }+\eta\left(v_{\max }-v_{\min }\right)
$$

where $v_{\min }$ is the minimum allowed/desired speed, $v_{\max }$ is the maximum allowed/desired speed and $\eta$ is a uniformly distributed random variable in the range $[0,1]$. The speed $v_{i}$ is selected once at the beginning of each trip and kept constant until the next destination is reached.

\section{Manhattan Model}

The Manhattan model [10] adds complexity to the speed management of the constant speed motion (CSM) model by updating the vehicles speed according to the following conditions.

$$
v_{\mathrm{i}}(\mathrm{t}+\triangle \mathrm{t})=\left\{\begin{array}{lr}
v_{\mathrm{i}+1}(\mathrm{t})-a / 2, & \text { if } \Delta \mathrm{x}_{\mathrm{i}}(\mathrm{t}) \leq \Delta \mathrm{x}_{\min } \\
\tilde{v}_{\mathrm{i}}(\mathrm{t}+\triangle \mathrm{t}), & \text { otherwise }
\end{array}\right.
$$




$$
\tilde{v}_{\mathrm{i}}(\mathrm{t}+\triangle \mathrm{t})=\min \left\{\max \left\{v_{\mathrm{i}}(\mathrm{t})+\eta a \triangle \mathrm{t}, v_{\min }\right\}, v_{\max }\right\}
$$

where $\eta$ is the same uniform random variable which was introduced before and $a$ is the vehicle's uniform acceleration. $\Delta x_{\min }$ and $\Delta x_{\max }$ are the minimum and maximum allowable inter-vehicle spacing, respectively. The Manhattan model thus, adds some acceleration-bounded randomness in the velocity update and from Equations (2) above, imposes speed limitation to prevent vehicles from overlapping [11]. The main drawback of this model is that it lacks the pause handling at road inter-sections.

\section{Freeway Model}

The Freeway model is designed for road topology graphs representing non-communicating, bi-directional, multi-lane freeways traversing the entire simulation area [12]. According to [9], the movement of each vehicle is restricted to the lane it is moving on, and the following speed management rules apply to vehicle $i$ :

- Speed update: The speed is varied by a random acceleration of maximum magnitude $a$. If a random variable $\eta$ is defined to be uniformly distributed in the range $[-1,1]$, then this rule can be expressed as

$$
v_{\mathrm{i}}(\mathrm{t}+\Delta \mathrm{t})=v_{\mathrm{i}}(\mathrm{t})+\eta \mathrm{a} \Delta \mathrm{t}
$$

- Speed bounding: At any particular moment during motion, the speed of a vehicle cannot be lower than a minimum value $\left(v_{\min }\right)$ and cannot exceed a maximum value $\left(v_{\max }\right)$. This constraint can be formulated as

$$
v_{\mathrm{i}}(\mathrm{t}+\Delta \mathrm{t})=\min \left\{\max \left[v_{\mathrm{i}}(\mathrm{t}+\Delta \mathrm{t}), v_{\min }\right], v_{\max }\right\}
$$

- Speed reduction: In order to avoid overlapping (collision situation) with the front vehicle, a minimum safety distance must be observed. This can formally be enforced as

$$
v_{\mathrm{i}}(\mathrm{t}+\triangle \mathrm{t})=\left\{\begin{array}{lr}
v_{\mathrm{i}+1}(\mathrm{t})-\mathrm{a} / 2, & \text { if } \Delta \mathrm{x}_{\mathrm{i}}(\mathrm{t}) \leq \Delta \mathrm{x}_{\text {min }} \\
\tilde{v}_{\mathrm{i}}(\mathrm{t}+\triangle \mathrm{t}), & \text { otherwise }
\end{array}\right.
$$

Each vehicle starts its movement at one end of a lane, with a speed that is initially selected as being uniformly distributed in the range $\left[v_{\min }, v_{\max }\right]$, and stops with it once it reaches the other extreme end of the same lane. Then a new movement is started on a randomly selected lane and the process is repeated. 


\subsection{Proposed Non-standard Vehicular Mobility Models}

Several non-standard vehicular mobility models have been proposed in the literature. However, we will only highlight a few key examples that are directly relevant to our work.

An analysis of improving data throughput and increasing the handover probability during network layer handovers is given in [13]. The proposed method aims at improving handover performance by maximising both the handover probability and data throughput in vehicular scenarios. Our current study is an extension of the method proposed in [13].

In [7], an enhanced network layer handoff performance meant to minimise the handoff failure probability in next generation wireless systems is proposed. Based on the information of false handoff probability, the authors analyse its effects on mobile speed and handoff signalling delay.

Authors of [14] propose a scheme meant to provide high accurate prediction of the next crossing cell that the $\mathrm{MN}$ is going to go, in order to avoid over-reservation of the limited system resources thereby, reducing wastage of such resources.

In [15], a method of minimising handoff latency by angular displacement method using Global Positioning System (GPS) based map is proposed. This is achieved by minimising the number of access points (APs) scanned by the mobile node $(\mathrm{MN})$ during each handover procedure.

A link layer assisted handover algorithm meant to enhance Received Signal Strength (RSS) value and thus, reduce the handover latency and handover failure is proposed in [16]. This algorithm employs an approach where access points used in a wireless LAN environment and a dedicated MAC bridge are jointly used to achieve packet loss without altering the Mobile IP specifications.

A new enhanced Handoff Protocol for Integrated Networks (eHPINs) which localizes the mobility management enabling fast handover is introduced in [17]. The eHPINs scheme alleviates the service disruptions during roaming in heterogeneous IP-based wireless environments thereby, improving the QoS for real-time applications in such networks.

In [18], a scheme called Simplified Fast Handover in Mobile IPv6 Networks (SFMIPv6) is proposed. This scheme is an enhancement of the FMIPv6 standard which significantly reduces the anticipation time for fast handovers thereby, increasing the probability of predictive fast handover execution. 


\section{Description and Modelling of the Proposed Scheme}

The access network of the proposed scheme is based on next generation wireless systems with radio base stations (BSs) providing Internet connectivity to on-board users. Furthermore, the proposed scheme adopts the concept of sectorisation by dividing the coverage area of a single BS into six cell sectors using direction antennas. In addition, we assume that the vehicle under investigation is mounted with a Global Positioning System (GPS) device to keep track of the real-time mobility parameters of the vehicle such as speed, position and direction of motion [7, 13].

\subsection{Handover and Non-handover Regions}

To increase the rate and accuracy of candidate router discovery, each cell sector is divided into two regions, which are: the handover region (near the cell border) and the non-handover region (near the transmitter). In the nonhandover region, the $\mathrm{MN}$ is under the full coverage of the serving $\mathrm{BS}$ and thus, there is no need for handover execution. However, when the MN enters the handover region, it experiences different BS signal probes with varying signal strength and quality which can trigger the handover process [19]. Figure 2 shows an example of handover and non-handover regions between for BSs belonging to two different subnets.

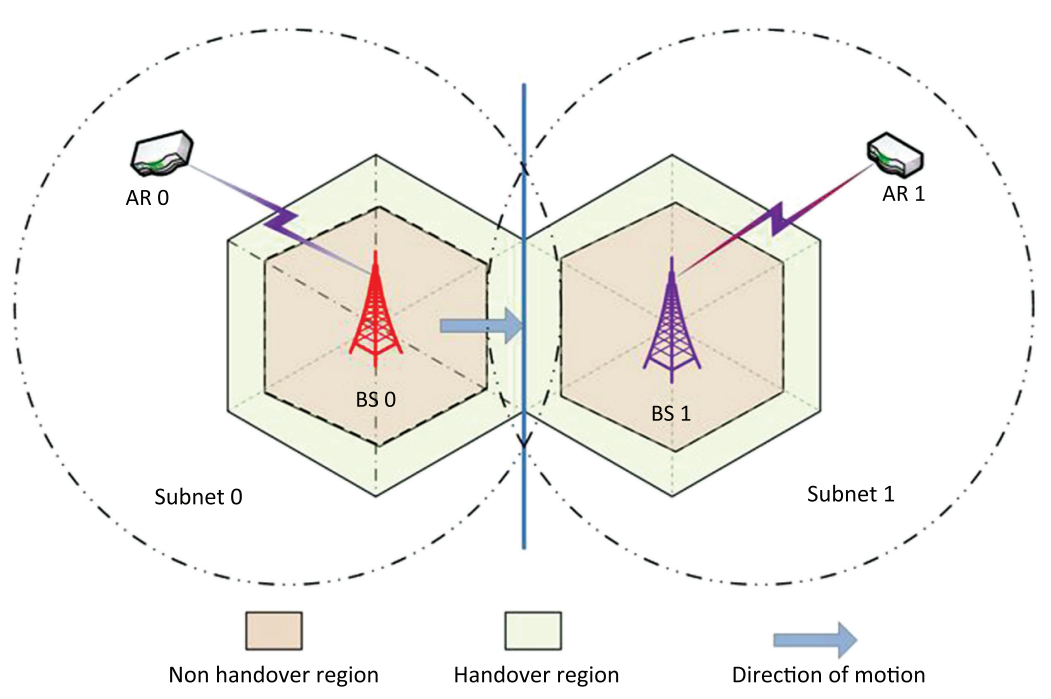

Figure 2 Handover and non-handover regions in the proposed scheme 
When a vehicle moves from the non-handover region to the handover region as shown by the arrow in the Figure 2, handover preparations start as the MN scans and observes potential candidate BSs through probes and pilot signals. In the BS probes are neighbour cell information such as network ID, subnet ID, BS ID and cell ID. In addition, the attached GPS device traces the position, speed and direction of the vehicle which are recorded and stored in the memory of the Application Units (AUs). The target cell information and the GPS stored information are transmitted to the current Access Router (cAR) via the current BS (cBS). During inter-subnet handover preparation, the $c A R$ requests the target Access Router (tAR) to reserve radio resources for the $\mathrm{MN}$ to use at the target $\mathrm{BS}$ (tBS). At the same time, a bi-directional tunnel is created between the two ARs and the cAR transfers data packets meant for the MN to the tAR.

\subsection{Cell Overlap Region}

The coverage area of a single BS is represented by a regular hexagon. For an inter-subnet handover process, we assume the two adjacent regular hexagons representing service areas of two neighbouring BSs to be overlapping $[7,18]$. Therefore, the near-to-reality circular cells do overlap thereby, creating a cell overlap region where handover execution takes place. Figure 3 shows the cell overlap region for two radio cells belonging to two different subnets.

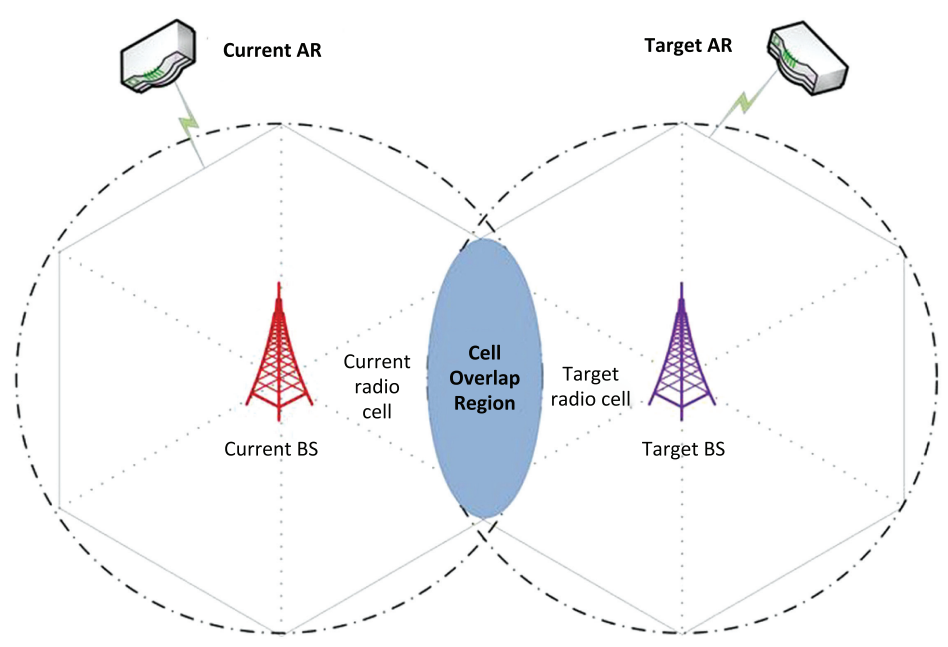

Figure 3 Cell overlap region for an inter-subnet handover scenario 


\subsection{Mobility Modelling}

From Figure 3 above, we geometrically formulate the mobility model of the vehicle as it enters the cell overlap region during an inter-subnet handover process. This is illustrated in Figure 4 below. $R S S_{T h}$ and $R S S_{\text {min }}$ depict the received signal strength (RSS) threshold value required to initiate a handover and the MN's minimum $R S S$ value needed to communicates successfully with a BS, respectively $[7,18]$. The distance travelled by the MN from the time $R S S_{T h}$ is detected to the time a handover takes place is given by $d$ with $\alpha$ being the angular direction of travel. We assume the vehicle under investigation enters the cell overlap region at point $B$ and moves in a straight line through the overlap region with an angular displacement of $\alpha \epsilon[-\theta, \theta]$ radians with respect to line $B I$ in Figure 4.

In the figure, when the vehicle crosses line $D G$, an inter-subnet handover occurs. This consists of link-layer handover from current BS to target BS followed by network-layer handover from current Access Router (AR) to target AR. By geometry, $E I=A I=r$, (i.e., one side of a regular hexagon = radius of the circle circumscribing the hexagon). Let $C O=\delta$ (i.e., size of intrusion of one hexagon into another).

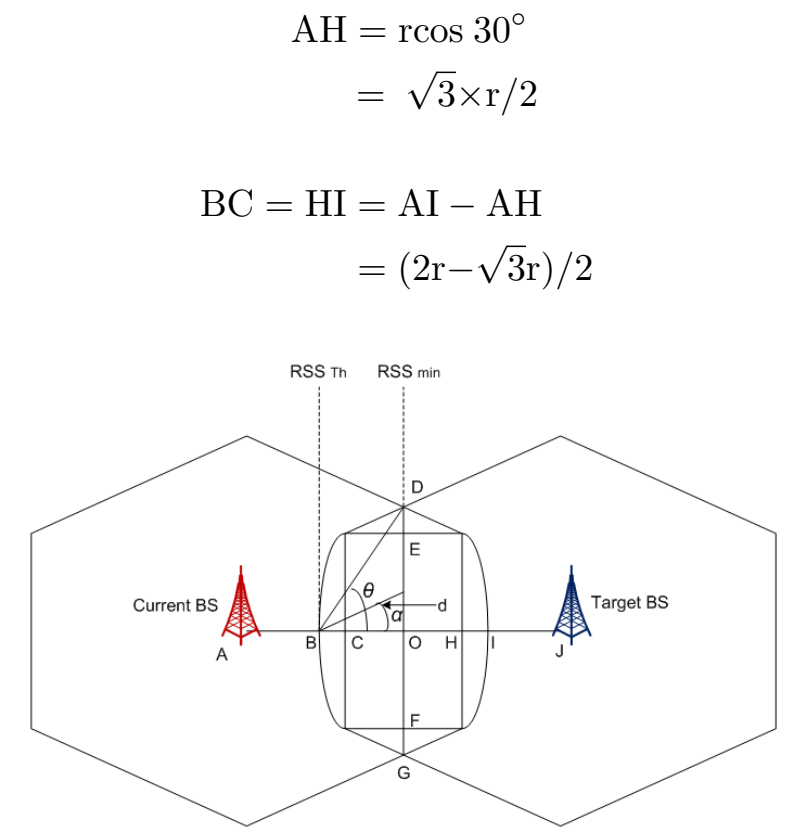

Figure 4 Handover mobility model analysis 
We assume that a handover takes place only when the vehicle crosses line $D G$ in Figure 4.

$$
\begin{aligned}
\mathrm{BO} & =\mathrm{BC}+\mathrm{CO} \\
& =(2 \mathrm{r}-\sqrt{3} \mathrm{r}+2 \delta) / 2
\end{aligned}
$$

Further, from the figure, we can deduce that

$$
\begin{aligned}
\mathrm{DE} & =\delta \tan 30^{\circ} \\
& =\delta / \sqrt{3} \\
\mathrm{DO} & =\mathrm{DE}+\mathrm{EO} \\
& =\frac{\delta}{\sqrt{3}}+\frac{\mathrm{r}}{2} \\
& =(2 \delta+\sqrt{3} \mathrm{r}) / 2 \sqrt{3} \\
\tan \theta & =\frac{\mathrm{DO}}{\mathrm{BO}} \\
& =\frac{\sqrt{3} \mathrm{r}+2 \delta}{\sqrt{3}(2 \mathrm{r}-\sqrt{3} \mathrm{r}+2 \delta)}
\end{aligned}
$$

\subsection{Correct Handover Initiation Probability}

If we let the initial speed of the vehicle to be $v_{i}$ at an initial angular displacement of $\theta_{i}$, then the direction of motion is a uniformly distributed random variable with the Probability Density Function (PDF) given by

$$
\mathrm{f}_{\theta}(\theta)=\left\{\begin{array}{lr}
1 / 2 \pi ; & -\pi \leq \theta \leq \pi \\
0 \quad ; & \text { otherwise }
\end{array}\right.
$$

Handover to the target radio cell occurs only if the vehicle's direction of motion from point $B$ in Figure 4 is in the range $(-\theta, \theta)$, where $\theta=\tan ^{-1}\left[\frac{\sqrt{3} \mathrm{r}+2 \delta}{\sqrt{3}(2 \mathrm{r}-\sqrt{3} \mathrm{r}+2 \delta)}\right]$. The probability of correct handover initiation $\left(P_{\text {correct }}\right)$ when the vehicle is at point $B$ is calculated as follows.

$$
\begin{aligned}
P_{\text {correct }} & =\int_{-\theta}^{\theta} f_{\theta}(\theta) d \theta \\
& =\frac{\theta}{\pi}
\end{aligned}
$$




$$
=\tan ^{-1}\left\{\frac{\sqrt{3} r+2 \delta}{\sqrt{3}(2 r-\sqrt{3} r+2 \delta)}\right\} / \pi
$$

Therefore, the probability of correct handover initiation is dependent on the value of both $\delta$ and $\mathrm{r}$. When $\delta=0$, probability of correct handover initiation has a constant value $\left(\mathrm{P}_{\text {correct }}=0.417\right)$. According to [7], handover failure probability increases as handover signalling delay increases. As a consequence, probability of handover success decreases as handover signalling delay increases. In Figure 4, for the direction of motion of the vehicle from $\mathrm{B}$, where $\alpha \epsilon[-\theta, \theta]$, the time taken $\mathrm{t}$, from the moment $R S S_{T H}$ is detected by the $\mathrm{MN}$ to the moment a handover process starts is given by

$$
t=(2 \mathrm{r}-\sqrt{3} \mathrm{r}+2 \delta) /(2 v \cos \alpha)
$$

where $v$ is the vehicle's constant speed. The PDF of the angular direction, $\alpha$ is given by

$$
F_{\alpha}(\alpha)= \begin{cases}\frac{1}{2 \theta_{1}} ; & \text { where }-\theta_{1}<\alpha<\theta_{1} \\ 0 ; & \text { otherwise }\end{cases}
$$

From Equation (11), $t$ is a function of $\alpha$ i.e., $t=y(\alpha)$ and therefore, (11) can be expressed as

$$
y(\alpha)=\frac{(2 r-\sqrt{3} r+2 \delta)}{2 v \cos \alpha}
$$

According to [7], we can calculate the PDF of $t$ as

$$
F_{t}(t)=\sum \frac{F_{\alpha}\left(\alpha_{i}\right)}{\left|y^{\prime}\left(\alpha_{i}\right)\right|}
$$

where $\alpha_{i}$ are the two roots of the equation $t=y(\alpha)$ in the interval $\left[-\theta_{1}, \theta_{1}\right]$. In either case, $F_{\alpha}\left(\alpha_{i}\right)=\frac{1}{2 \theta_{1}}$, for $i=1$ and 2 . Consequently, $F_{t}(t)$ can be expressed as

$$
F_{t}(t)=\frac{1}{\theta_{1}\left|y^{\prime}\left(\alpha_{i}\right)\right|}
$$

where $y^{\prime}(\alpha)$ is the first derivative of $y(\alpha)$ and is given by

$$
\begin{aligned}
y^{\prime}(\alpha) & =t \tan \alpha \\
& =t \sqrt{\left(\sec ^{2} \alpha-1\right)}
\end{aligned}
$$




$$
=t \sqrt{\left\{\left(\frac{2 v t}{2 r-\sqrt{3} r+2 \delta}\right)^{2}-1\right\}}
$$

From Equations (15) and (16), the PDF of $t$ becomes

$$
F_{t}(t)=\left\{\begin{array}{lr}
\frac{2 r-\sqrt{3} r+2 \delta}{\theta_{1} t \sqrt{\left[(2 v t)^{2}-(2 r-\sqrt{3} r+2 \delta)^{2}\right]},}, & \Phi_{1}<\Phi_{2} \\
0, & \text { otherwise }
\end{array}\right.
$$

where $\Phi_{1}=\frac{2 r-\sqrt{3} r+2 \delta}{2 v}$ and $\Phi_{2}=\frac{\sqrt{\frac{(2 r-\sqrt{3} r+2 \delta)^{2}}{4}+\frac{(\sqrt{3} r+2 \delta)^{2}}{12}}}{v}$.

According to [7, 15], if $\vartheta$ is the handover signalling delay such that $P(t<\vartheta)$ is the probability that $t<\vartheta$, then the probability of false handover initiation, $P_{F a l s e}$ is given by

$$
P_{\text {False }}\left\{\begin{array}{lc}
0, & \vartheta<\Phi_{1} \\
P(t<\vartheta), & \Phi_{1}<\vartheta<\Phi_{2} \\
1, & \vartheta>\Phi_{2}
\end{array}\right.
$$

For the range of values of $\vartheta$ in the interval $\vartheta_{1}=\Phi_{1}<\vartheta<\vartheta_{2}=\Phi_{2}$ and using Equation (18), we obtain an expression for $P(t<\vartheta)$ as

$$
\begin{aligned}
P(t<\vartheta) & =\int_{t}^{\vartheta} F_{t}(t) d t \\
& =\int_{\vartheta_{1}}^{\vartheta_{2}} \frac{2 r-\sqrt{3} r+2 \delta}{\theta_{1} t \sqrt{\left[(2 v t)^{2}-(2 r-\sqrt{3} r+2 \delta)^{2}\right]}} d t \\
& =\frac{1}{\theta_{1} \cos \vartheta\left[\frac{2 r-\sqrt{3} r}{2 v \vartheta}\right]}
\end{aligned}
$$

From Equations (18) and (19), we get the relationship between correct handover probability $\left(P_{\text {correct }}\right)$ and vehicle's speed $v$, as follows.

$$
P_{\text {correct }}\left\{\begin{array}{lrl}
0, & \vartheta & >\Phi_{2} \\
1-P(t<\vartheta), & \Phi_{1}<\vartheta<\Phi_{2} \\
1, & \vartheta & <\Phi_{1}
\end{array}\right.
$$




\subsection{Cell Overlap Crossing Time and Data Throughput}

For a given vehicle $V$, traversing an area covered by a wireless cell $C$ at an average speed $v$, the cell crossing time of $V$ through $C$, denoted by $\Delta T$, is the overall time that $V$ can spend under the coverage area of $C$ [20]. From Figure 4, we can calculate the cell crossing time of the cell overlap region as follows. We assume the vehicle's trajectory follows a Manhattan mobility model and is constrained by straight lane roads. Let $t_{\text {in }}$ and $t_{\text {out }}$ denote the times at which vehicle enters the cell overlap region and the time at which it leaves the cell overlap region, respectively. The cell overlap crossing time is therefore, given by

$$
\begin{aligned}
\Delta \mathrm{T} & =\mathrm{t}_{\text {out }}-\mathrm{t}_{\text {in }} \\
& =(2 r-\sqrt{3} r+2 \delta) /(v \cos \alpha)
\end{aligned}
$$

where $v$ is the vehicle's average speed. Therefore, the cell overlap region crossing time, $\Delta T$ is dependent on $r, \delta, \alpha$ and $v$.

Given the above assumptions and definitions, we can model the data throughput, $\gamma$ that the MN would experience by traversing through the cell overlap region during the period, $\Delta T$ as a function of the system bandwidth $B W$, which is assumed to be constant during the period $\Delta T$. According to [20], the throughput experienced by the MN moving in the region comprising heterogeneous access radio cells during the period $\Delta T$ is a positive range function $\gamma: \mathfrak{R} \rightarrow \mathfrak{R}^{+}$defined as

$$
\gamma=\rho\left(\mathrm{B}_{\mathrm{CN}}-\eta\right)\left(\triangle \mathrm{T}-\mathrm{T}_{\mathrm{L}}\right)+(1-\rho) \mathrm{B}_{\mathrm{SN}} \triangle \mathrm{T}
$$

where $\rho$ is an indicator function such that $\rho=1$ when vertical handover is executed and zero otherwise, $T_{L}$ is the handover latency, $B_{C N}$ is the bandwidth of the candidate network, $B_{S N}$ is the bandwidth of the serving network and $\eta \in \mathfrak{R}^{+}$is the hysteresis factor introduced to avoid vertical handover occurrence when two competing networks have negligible bandwidth difference. In our model, we consider an intra-network handover scenario, hence a horizontal handover process. In this case, $\rho=0$ and $B_{C N}=B_{S N}=B W$. The throughput due to the current $\mathrm{BS}$ is therefore given by

$$
\begin{aligned}
\gamma & =\triangle \mathrm{T} \cdot B W \\
& =\{(2 r-\sqrt{3} r+2 \delta) B W\} /(v \cos \alpha)
\end{aligned}
$$

where $v$ is the average speed of the vehicle. 


\section{Simulations and Performance Analysis}

\subsection{Simulations Methodology}

The implementation and simulations methodology are hereby presented in this section. Simulation input parameters used in the performance analysis are given in Table 1.

Simulations were conducted in the MATLAB numerical analysis tool environment. In the analysis, a scenario was considered where an IP capable $\mathrm{MN}$ moves from one subnet to another in a straight line at various average speeds in the range $(0-70 \mathrm{~m} / \mathrm{s})$. This is a typical range of speeds for most highway scenarios.

\subsection{Performance Analysis}

The performance evaluations were carried out in two phases. Firstly, we investigate the influence of $R S S_{T H}$ position (hence, cell intrusion distance $(\delta)$ ) and vehicle's average speed $(v)$ on probability of correct handover initiation $\left(P_{\text {correct }}\right)$. Secondly, we study the effects of vehicle speed $(v)$ and direction $(\alpha)$ on data throughput $(\gamma)$ during inter-subnet handovers.

\subsubsection{Probability of Correct Handover Initiation $\left(\boldsymbol{P}_{\text {correct }}\right)$ Analysis}

1. Effects of $R S S_{T H}$ Position on Probability of Correct Handover Initiation

From Equation (10) representing the probability of correct handover initiation $\left(P_{\text {correct }}\right)$, we can infer that if we unnecessarily increase the value of the cell intrusion distance $(\delta), P_{\text {correct }}$ decreases. This results in wastage of the limited wireless system resources. Moreover, this increases the network load that arises due to handover initiation. Figure 5 shows the relationship between $P_{\text {correct }}$ and $\delta$ for different cell sizes, ' $r$ '. The figure shows that, for a particular value of $r, P_{\text {correct }}$ decreases as $\delta$ increases. It can also be seen that the

Table 1 Simulation input parameters

\begin{tabular}{lll}
\hline Parameter & Symbol & Range of Values \\
\hline Cell intrusion distance & $\delta$ & $0-100 \mathrm{~m}$ \\
Vehicle's average speed & $v$ & $0-70 \mathrm{~m} / \mathrm{s}$ \\
Vehicle's angular direction & $\alpha$ & {$[-\pi, \pi] \mathrm{rad}$} \\
Network bandwidth & $B W$ & $0.5-2.0 \mathrm{Mbps}$ \\
Cell radius & $r$ & $1-10 \mathrm{Km}$ \\
Handover signalling delay & $\vartheta$ & $1-3 \mathrm{sec}$ \\
\hline
\end{tabular}




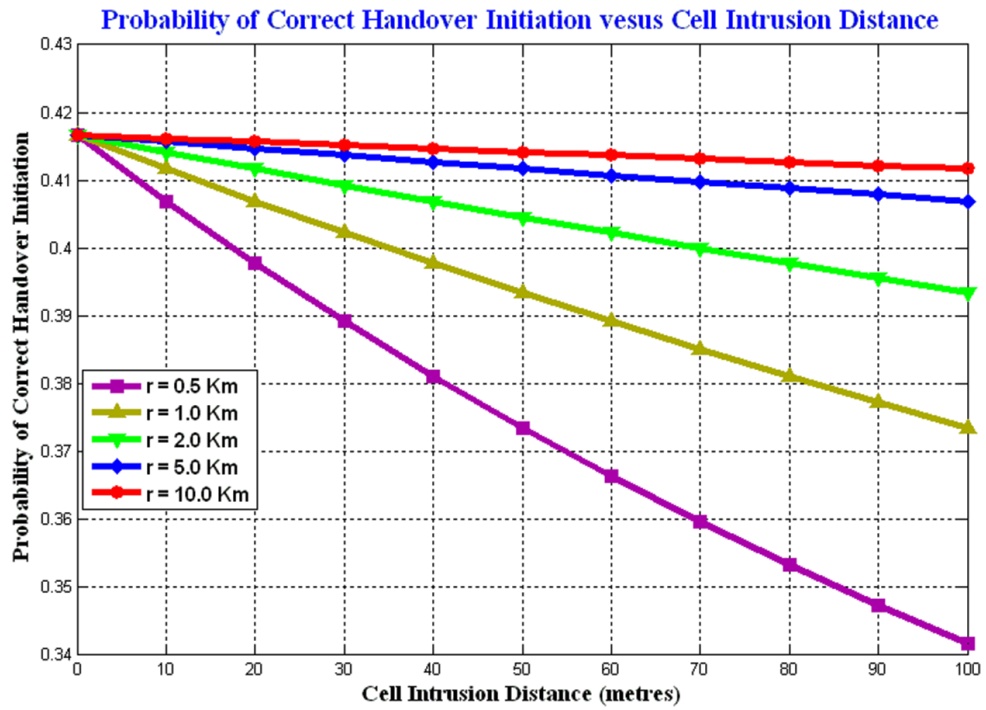

Figure 5 Relationship between $\left(P_{\text {correct }}\right)$ and cell intrusion distance $(\delta)$

problem of incorrect handover initiation becomes more and more pronounced when cell size decreases. Our main target in this respect is to increase $P_{\text {correct }}$. For this reason, we have to adjust the value of $\delta$ in such a way that $P_{\text {correct }}$ will be highest. In order to get a constant value of $P_{\text {correct }}$, we let $\delta=0$, for which we get the value $P_{\text {correct }}=0.417$.

\section{Effects of Vehicle Speed on Probability of Correct Handover Initiation}

From Equation (20), we can infer that when $\frac{2 r-\sqrt{3} r+2 \delta}{2 v}<\vartheta<$ $\frac{\sqrt{\frac{(2 r-\sqrt{3} r+2 \delta)^{2}}{4}+\frac{(\sqrt{3} r+2 \delta)^{2}}{12}}}{v}$, for a fixed value of $R S S_{T H}$ (and hence a fixed value of corresponding $\delta$ ), the probability of correct handover initiation $\left(P_{\text {correct }}\right)$ depends on the vehicle's speed. In fact, the probability of correct handover initiation decreases with increase in speed. That is, $P_{\text {correct }}$ is inversely related to vehicle speed. If speed is $v$, then we can write: $P_{\text {correct }} \propto \frac{1}{v}$. We testify this inverse proportionality with the help of a simulation. In our simulation, we considered a cell $(r=3 \mathrm{~km})$ and the handover signalling delay $(\vartheta=3 \mathrm{sec})$. Figure 6 shows the relationship between probability of correct handover initiation and vehicle's speed for an inter-subnet handover process. In the figure, numerical values of $P_{\text {correct }}$ for different values of $\delta$ (delta) which correspond to different values of $R S S_{T H}$ positions are shown. Simulation results show that for a particular value of $\delta$, as speed increases, $P_{\text {correct }}$ 


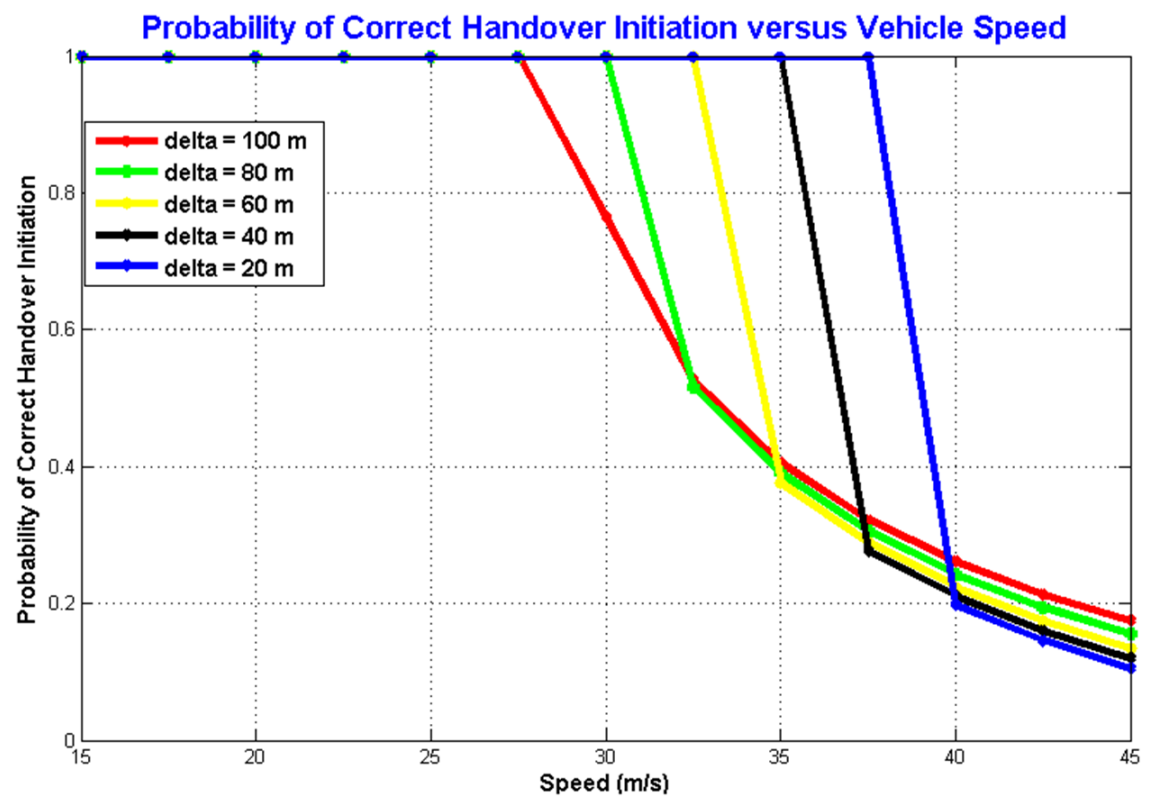

Figure 6 Relationship between $P_{\text {correct }}$ and vehicle speed

decreases since the vehicle requires less time to move out of the coverage range of the current BS into the coverage range of the target BS. Moreover, for a particular value of $R S S_{T H}$ position, $P_{\text {correct }}$ becomes less when handover signalling delay is increased. This is usually the case for inter-subnet and intersystem handovers. On the other hand, intra-subnet handovers experience less signalling delays with typical values of less than 1 second in cellular networks such as UMTS systems [7, 15]. Therefore, for inter-subnet handovers to experience increased $P_{\text {correct }}, \vartheta$ must be reduced to values less than 1 second. However, this comes at a price of inaccurate IP configurations and registration processes.

\subsubsection{Data Throughput $(\gamma)$ Analysis}

\section{Effects of Vehicle Speed on Data Throughput}

The relationship between data throughput, $\gamma$ and vehicle speed, $v$ is represented by Equation (23). From the equation, it can be deduced that throughput decreases as the average speed increases while the vehicle moves across the cell overlap region. Figure 7 shows the relationship between throughput due to current BS and vehicle's average speed for different cell sizes, ' $r$ ' 


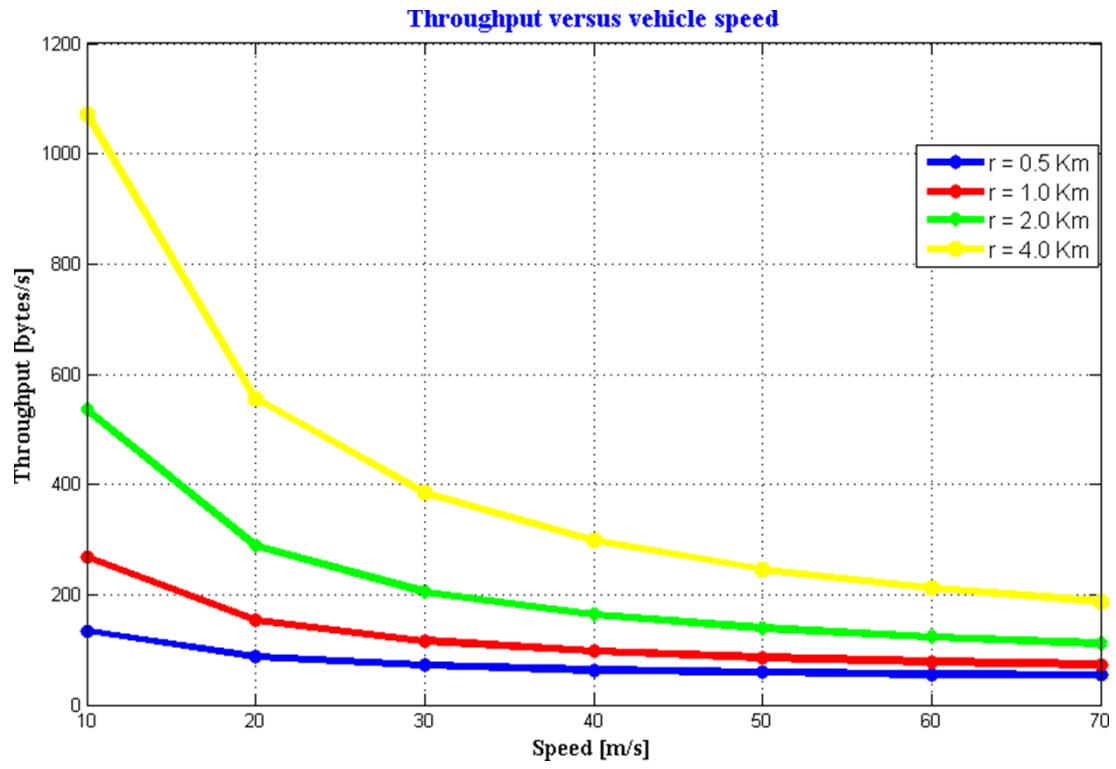

Figure 7 Relationship between data throughput and vehicle speed

at a constant network bandwidth of $2 \mathrm{Mbps}$. From the figure, it can be observed that for a single $\mathrm{MN}$ at a given speed, throughput is higher in a relatively bigger cell than a smaller cell. This is so because increasing cell size results in increased cell overlap region due to increased transmit power. Consequently, the $\mathrm{MN}$ takes longer time to handover when the serving radio cell is enlarged. To this end, we can state that increase in cell radius results in increased throughput. However, increased cell radius also results in increased interference from neighbour cells and this could compromise the network quality. Therefore, cell dimensioning should be carefully done so as to provide increased throughput while enjoying better network quality.

\section{Effects of Vehicle Direction on Data Throughput}

From Equation (23), it can be deduced that as the vehicle's angular direction, $\alpha$ is varied, the MN's data throughput, $\gamma$ due to current BS varies. When $\alpha$ is increased with respect to line BI in Figure $4, \gamma$ increases since the vehicle spends more time within the coverage area of the current BS before it completely moves to the coverage area of the target BS. Numerical results depicting the relationship between data throughput and vehicle direction are 


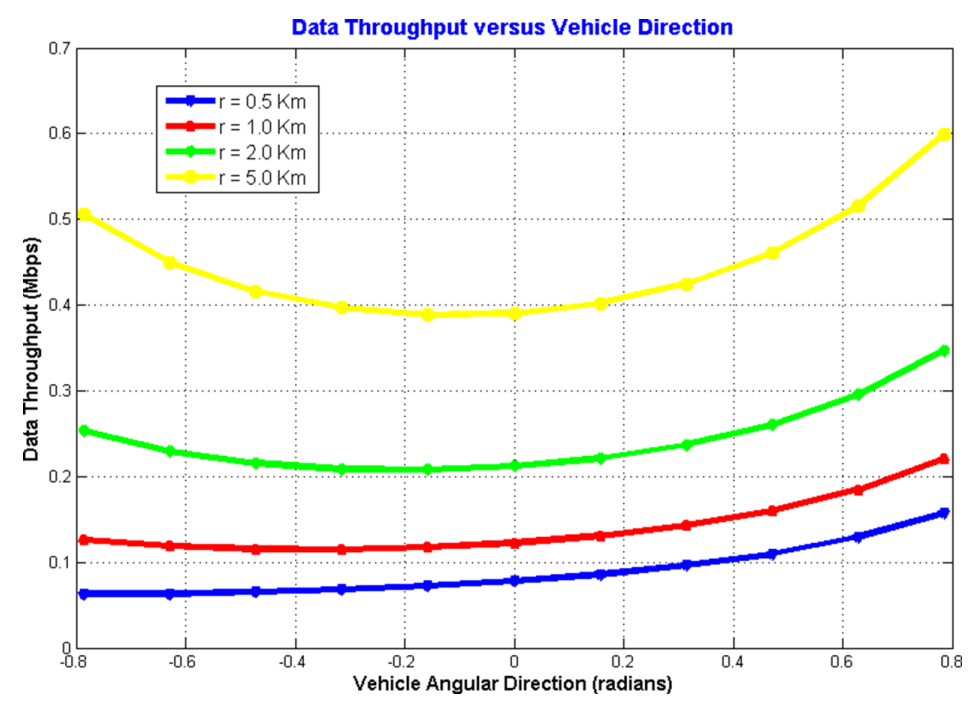

Figure 8 Relationship between data throughput and vehicle direction

shown in Figure 8. During simulations, the vehicle speed was fixed at a constant value of $45 \mathrm{~m} / \mathrm{s}$ which is a typical highway speed. Numerical results show that throughput increases with cell size of the current BS for a particular value of $\alpha$. However, typical cell sizes in heterogeneous access networks are in the range of $1-3 \mathrm{~km}[2,7,14]$. Therefore, careful dimensioning of cell radii and positioning of $R S S_{T H}$ (hence, $\delta$ value) must be taken into account during planning and designing of IP-based vehicular networks deploying throughput-sensitive applications.

\section{Conclusions and Future Work}

In this work, we introduced vehicular networks as an emerging wireless communication technology developed under the Intelligent Transportation Systems (ITS) to provide safety and non-safety related applications. We identified the negative effects of large signalling delays on throughputsensitive and delay-intolerant ITS applications during inter-subnet handovers. This formed the motivation for this paper which led to the proposal of a mobility scheme aimed at reducing the signalling delay and increasing the data throughput by maximising the probability of correct handover initiation. Through numerical analysis, it is observed that for a fixed cell size value, the probability of correct handover initiation decreases as the 
value of $R S S_{T h}$ position (hence, length $\delta$ ) increases. Furthermore, when a fixed value of $R S S_{T h}$ position is used, the probability of correct handover initiation decreases as vehicle speed increases. Based on this analysis, we suggest a method by which probability of correct handover initiation can be maximised and kept within constant limits. Furthermore, numerical analysis showed that data throughput decreases as vehicle speed increases. Moreover, data throughput increases with increase in vehicle's angular direction with respect to the shortest between the current BS and target BS during inter-subnet handovers. This gave us an insight on how cell size dimensioning can impact on data throughput and how a trade-off between data throughput and network quality has to be met during network planning and designing.

Future work should consider inter-Radio Access Technology (RAT) handovers comprising various heterogeneous access networks having different QoS demands and non-uniform coverage footprints. Furthermore, the proposed mobility model can be extended to standard vehicular network solutions such as the Wireless Access for the Vehicular Environment (WAVE) protocol stack for short-and medium-range V2V and V2I communications.

\section{References}

[1] S. Cespedes, X. Shen, C. Lazio, 'IP Mobility Management for Vehicular Communication Networks: Challenges and Solutions,' IEEE Communications Magazines, vol. 49, no. 5, pp. 187-194, May 2009.

[2] L. Banda, M. Mzyece, G. Noel, 'IP Mobility Support Solutions for Vehicular Networks,' IEEE Vehicular Technology Magazine (VTM), vol. 7, no. 4, pp. 77-87, Dec. 2012.

[3] Car-to-Car Communication Consortium, 'C2C-CC vehicular network architecture,' available at: http://www.car2car.org, 2011.

[4] V. Vassiliou, Z. Zinonos, 'An Analysis of Handover Latency Components in Mobile IPv6,' Journal of Internet Engineering, vol. 3, no. 1, pp. 230-240, 2009.

[5] L. Banda, M. Mzyece, G. Noel, 'An Enhanced FMIPv6 Scheme for Intersubnet Handovers,' Southern Africa Telecommunication Networks and Applications (SATNAC), Spier Wine Estate, Western Cape, South Africa, 1-4 Sept. 2013.

[6] A. Chen, C. Wu, J. Ho, 'Secure transparent Mobile IP for intelligent transportation systems,' IEEE International Conference on Networking, Sensing and Controls, Taipei, Taiwan, 2004. 
[7] D. Sarddar, et. al., 'Minimization of Handoff Failure Probability for NextGeneration Wireless Systems,' International Journal of Next-Generation Networks (IJNGN), vol. 2, no. 2, pp. 36-51, June 2010.

[8] M. M. Zonoozi, P. Dassanayake, 'User mobility modelling and characterisation of mobility patterns,' IEEE Journal on Selected Areas in Communication, vol. 5, no. 4, pp. 1239-1252, April 2011.

[9] S. Olariu, M. C. Weigle, 'Vehicular Networks: From Theory to Practice,' $1^{\text {st }}$ ed., Taylor \& Francis Group, Boca Raton, FL, 2009.

[10] F. Bai, N. Sadagopan, A. Helmy, 'The IMPORTANT framework for analysing the Impact of Mobility on Performance Of RouTing protocols for Ad-hoc NeTworks,' AdHoc Networks Journal-Elsevier Adhoc Networks, vol. 1, pp. 383-403, 2003.

[11] M. Fiore, J. Harri, 'The Networking Shape of Vehicular Mobility,' ACM MobiHoc, pp. 108-119, New York, NY, USA, Oct. 2007.

[12] M. Fiore, 'Mobility Models in Inter-Vehicle Communication Literature,' Tech. Rep., Nov. 2006 [Online]. Available: www.tlc-net works.polito.it/fiore/papers.

[13] L. Banda, M. Mzyece, G. Noel, 'An Analysis of Handover Probability and Data Throughput in Vehicular networks,' PACT 2013, Lusaka, Zambia, Jul. 2013.

[14] M. Al-Sanabani, et. al., 'Mobility Prediction Based Resource Reservation for Handoff in Wireless Cellular Networks,' International Arab Journal of Information Technology, vol. 5, no. 2, pp. 162-169, April 2008.

[15] D. Sarddar, et. al., 'Minimization of Handoff Latency by Angular Displacement Method using GPS Based Map,' International Journal of Computer Science Issues (IJCSI), vol. 7, no. 3, pp. 29-37, May 2010.

[16] H. Yokota, et. al., 'Link-layer assisted mobile IP fast handoff method over wireless LAN networks,' $8^{\text {th }}$ Annual International Conference on Mobile Computing and Networking, pp. 131-139, 2002.

[17] C. Makaya, S. Pierre, 'Enhanced Fast Handoff Scheme for Heterogeneous Wireless Networks,' International Journal of Computer Communications, pp. 2016-2029, Jan. 2008.

[18] N. V. Hanh, S. Ro, J. Ryu, 'Simplified fast handover in mobile IPv6 networks,' Computer Communication, vol. 31, pp. 3594-3603, June 2008 . 
[19] L. Banda, M. Mzyece, G. Noel, 'Fast handovers without DAD using Sector-based Vehicular Mobile IPv6,' Southern Africa Telecommunication Networks and Applications (SATNAC), East London, South Africa, 4-7 Sept. 2011.

[20] F. Esposito, et. al., 'On Modelling Speed-Based Vertical Handovers in Vehicular Networks-Dad, slow down, I am watching a movie,' Annual IEEE Global Telecommunications Conference (GLOBE-COM '10), Miami, FL, USA, Dec. 2010.

\section{Biographies}

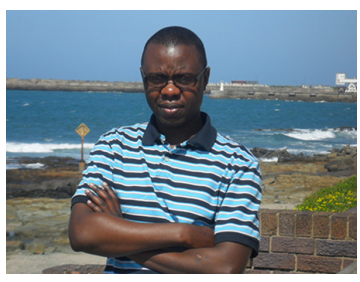

Laurence Banda received his BEng degree in electrical and electronic engineering from the University of Zambia (UNZA), Zambia in 2006, MSc degree in electronic engineering from ESIEE-Paris, France in 2011 and MTech degree in telecommunications engineering from Tshwane University of Technology (TUT), South Africa in 2012. He is currently working for Huawei Technologies, South Africa as a Wireless Trainer on LTE RNP and RNO products. His research interests include: vehicular networks, TCP/IP networks, 4G and beyond wireless broadband networks. (E-mail:laurencebandad@gmail.com).

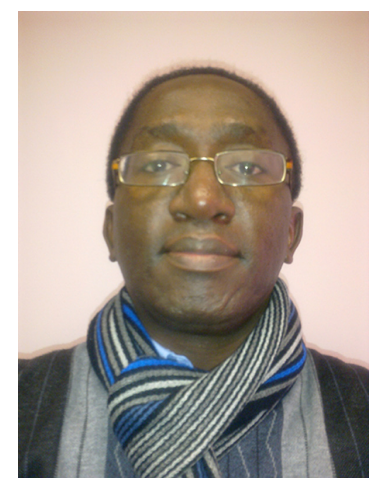

Mjumo Mzyece is an associate professor with the French South African Institute of Technology (FSATI) and the Department of Electrical Engineering 


\section{L. Banda and M. Mzyece}

at Tshwane University of Technology (TUT), Pretoria, South Africa. He received a BEng (Honours) in electronic and electrical engineering from the University of Manchester, England, and an MSc (Distinction) in communications technology and policy and a $\mathrm{PhD}$ in electronic and electrical engineering, both from the University of Strathclyde, Scotland. (E-mail: mzyecem@tut.ac.za). 